\title{
Intraosseous Compound-type Dermoid Cyst of the Jaw
}

\author{
${ }^{1}$ Dror M Allon, ${ }^{1}$ Shlomo Calderon, ${ }^{2}$ llana Kaplan \\ ${ }^{1}$ Department of Oral and Maxillofacial Surgery, Rabin Medical Center, Petach Tiqva and Goldschleger School of Dental Medicine \\ Tel-Aviv University, Israel \\ ${ }^{2}$ Institute of Pathology, Tel-Aviv Souraski Medical Center and Sackler School of Medicine, Tel-Aviv University, Israel
}

Correspondence: Ilana Kaplan, Director of Oral Pathology Services, Tel-Aviv Souraski Medical Center, 6 Weizman St. Tel-Aviv, Israel, e-mail: ig_kaplan@012.net.il

\begin{abstract}
Dermoid cysts within the maxillofacial skeleton are extremely rare, with only 13 cases in the literature. The present report describes a new long-standing intraosseous DC and reviews the literature.

A 69-year-old female presented with an expansile mandibular lesion, presenting a large radiolucency with the second and third molars impacted and displaced to the inferior mandibular border. Microscopic examination showed an intraosseous DC, lined by stratified keratinizing epithelium, with multiple aggregates of sebaceous cells and well formed sebaceous glands. Impaction of both second and third molars is an indication of initial development before the physiologic eruption time at approximately age 12 . Thus, this presents an extremely longstanding intraosseous DC of at least 45 years duration. Analysis of the literature indicates that in comparison with the more common odontogenic keratocyst, intraosseous DC seems to have a very slow growth rate, and an indolent behavior.

Keywords: Intraosseous, dermoid cyst, mandible.
\end{abstract}

\section{INTRODUCTION}

The term dermoid cyst is used to describe three closely related cystic lesions: epidermoid cyst, dermoid cyst, and teratoma. The epidermoid or simple type is characterized by a stratified keratinizing epithelial lining resembling epidermis, devoid of skin appendages. The dermoid (compound) type has a lining similar to that of the epidermoid type, but contains skin appendages such as hair follicles, sebaceous or sweat glands. Teratomas (or complex type) include other tissue types of both mesodermal and endodermal origin, such as cartilage, muscle, or nerve. ${ }^{1}$

The most common sites of dermoid cysts are in the soft tissues of the head and neck. In the oral cavity DC usually presents as a solitary, painless, midline mass in the floor of the mouth or the submandibular region. ${ }^{1,2}$ Less frequently in other intraoral locations, such as the lateral and anterior tongue, buccal mucosa, and lower lip. Intraosseous DC (IDC) is extremely rare in the maxillofacial region. Only 13 such cases have been documented in the literature, 7 in the mandible and 6 in the maxilla. ${ }^{3-11}$

In the present report, we describe a case of long-standing intraosseous compound-type dermoid cyst in the angle of the mandible. In addition, we review the literature and compare the features of dermoid cyst with odontogenic keratocyst of the jaw.

\section{CASE REPORT}

A 69-year-old woman presented with lingual and buccal expansion of the mandible in the left molar region. The height of the alveolar crest was increased, with ulceration of the overlying mucosa caused by trauma from opposing teeth. Friable erythematous tissue surrounded the periphery of the ulcer and the second and third molars were not present in the arch. The remaining mucosa was intact. The patient reported mild dull pain, consistent with the ulcer. Sensory function of the mandibular and mental nerves was normal. Lymph nodes were not palpable. The patient's medical history was noncontributory. A panoramic radiograph taken 4 years earlier showed a large unilocular radiolucency with 2 impacted molars displaced towards the lower border, and inferior displacement of the alveolar canal. Neither biopsy nor other treatment had been performed at that time. In a repeated radiograph further displacement of the teeth and the alveolar canal were observed, however, the lower border and alveolar crest were intact. A dental computed tomography $(\mathrm{CT})$ scan demonstrated a $4 \times 3.1 \mathrm{~cm}$ expansile 
lytic lesion in the left mandible, with thin cortical plates, and focal perforation of the medial cortical plate inferior to the level of the apices (Figs 1A and B).

Differential diagnosis included odontogenic keratocyst, central giant cell granuloma and ameloblastoma. Treatment consisted of excision of the gingival ulcer and surrounding tissue via an intraoral approach, and enucleation of the cyst with the impacted teeth. During enucleation, a large amount of white, soft, cheese-like material was found inside the lumen. The cyst was separated from oral mucosa by intact bone.
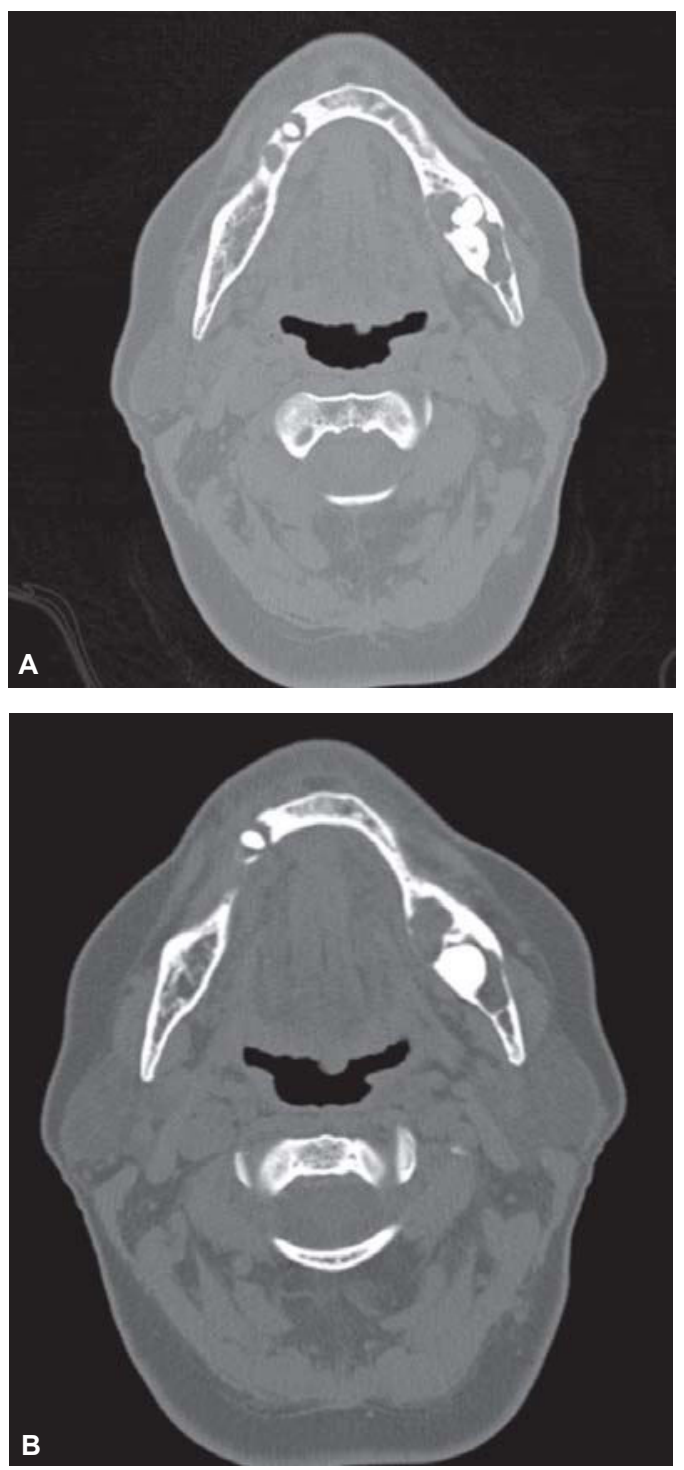

Figs 1A and B: CT scan, axial view at the level of the angle of the mandible. Expansion is evident, with thinning of the cortical plate on the buccal aspect and cortical perforation on the lingual aspect. Within the lesion the crown of an impacted tooth is protruding
On histopathological examination, two distinct lesion were identified. The sections from the superficial areas showed a peripheral giant cell granuloma with ulcerated overlying mucosa. The sections from the intraosseous lesion showed a cyst lined by stratified keratinizing epithelium. Multiple aggregates of sebaceous cells, and well-formed lobules of sebaceous glands were present in the cyst wall, with strands of keratin in the lumen. The adjacent connective tissue was fibrous, cellular, and free of inflammation in all examined sections (Figs 2 and 3). The final diagnosis was intraosseous DC (IDC).

Three years after surgery, complete bony regeneration was noted with no evidence of recurrence.

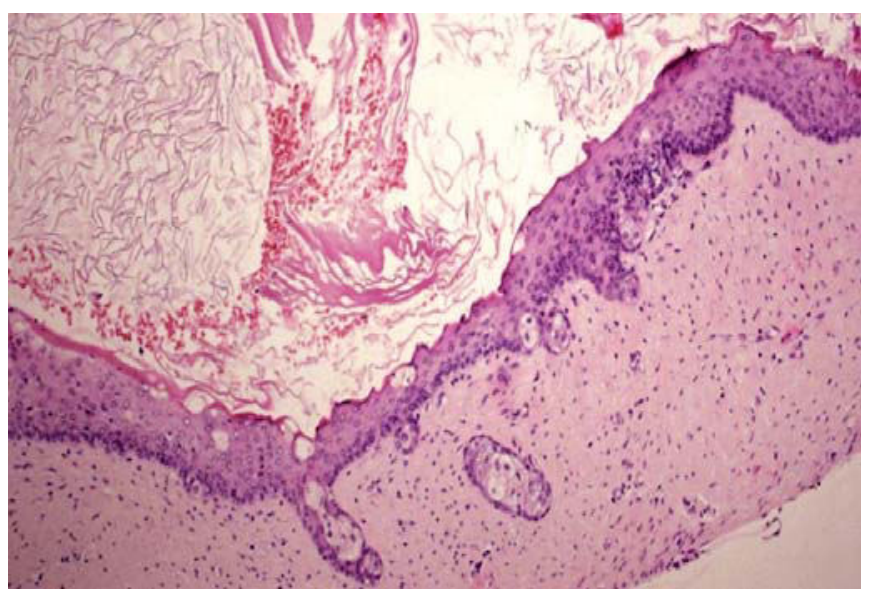

Fig. 2: Histopathologic section showing the keratinizing squamous epithelial cyst lining, with both aggregates of sebaceous cells within the lining, and well-formed sebaceous glands in the underlying cyst wall. Inflammation is absent. The lumen is filled with "onion skin" keratin (H\&E, original magnification X100, inset X 200 )

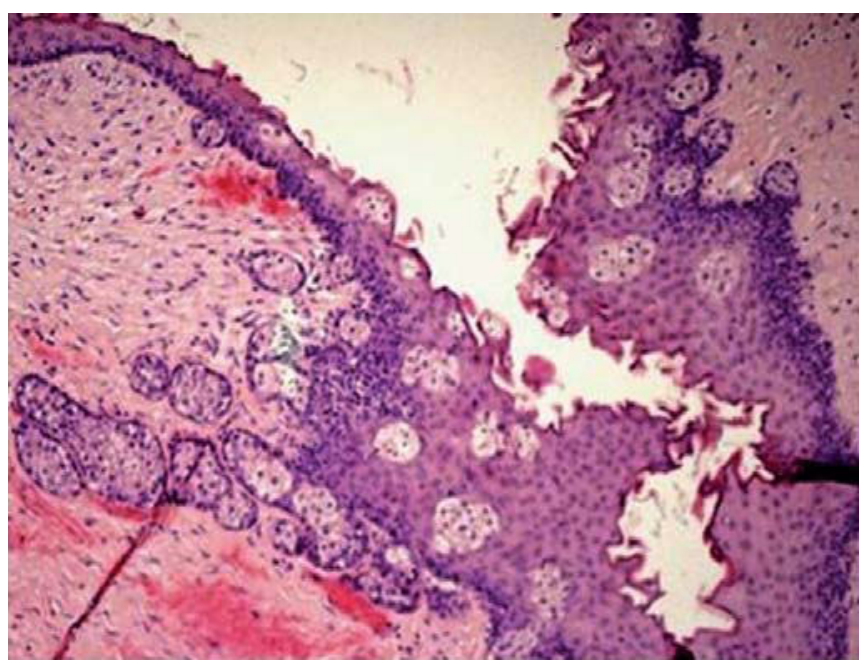

Fig. 3: In a different area of the lining, well-formed sebaceous glands are abundant. The underlying connective tissue is cellular, collagenized and free of inflammation (H\&E, original magnification X100) 


\section{LITERATURE REVIEW}

A search of the English-language literature 1966 to 2009 yielded 13 cases of true IDC of the jaws. The present case brings the total to 14 cases, with an age range of 5 to 69 years (mean 32) and a M:F ratio of 1:1.5. Six cases occured in the maxilla, most frequently in hard palate and incisorcanine area, 8 in the mandible, most frequently in the molar area.

All cases presented a round/oval well-defined unilocular radiolucency, with lobulated borders in one case. A diameter of 10 to $60 \mathrm{~mm}$ (clinically or by CT scan) was reported. Cortical expansion, tooth impaction and displacement of the alveolar canal were reported in 3 cases each (21\%), without sensory impairment.

Histopathologically, keratinizing squamous epithelium lining with sebaceous elements were typical in all cases. In 4 cases (27\%), sweat glands or hair follicles were reported. White cheese-like content was described in 10 cases. Followup ranged from 5 to 36 months, without recurrence in any of the patients.

\section{DISCUSSION}

IDC is one of the rarest types of cyst of the jawbone, with a poorly understood origin. One theory explaining the origin of jawbone IDC suggested embryonic ectoderm entrapped in the developing mandible (although not along embryonic clefts, which are not present in the developing mandible), which maybe induced to proliferate by local (dental) inflammation. Skin appendages in some IDC support this theory. ${ }^{4}$ Fordyce's granules (ectopic sebaceous glands in oral mucosa), present in $80 \%$ of the population, may also be the origin, by migration of oral epithelium into bone through a fistula or following marsupialization procedure. ${ }^{1,12}$

Finally, undifferentiated odontogenic epithelium in dentigerous cysts was suggested to undergo sebaceous differentiation by induction of the mesodermal organizer factors. ${ }^{1}$ However, this mechanism fails to explain the presence of sweat glands and hair follicles separate from the lining epithelium.

In the present case it is highly unlikely the sebaceous glands originated from Fordyce's granules, since there was intact bone between the cyst and the oral mucosa. The cortical perforation found on the CT scan was below the level of the floor of the mouth; absence of inflammation in the cyst wall also indicated no communication between cyst and mucosa.
There is histopathological resemblance between the simple type of DC and the much more common odontogenic keratocyst (OKC). In OKC, the epithelial lining is thin $(<5$ to 8 cells), covered by a thin corrugated parakeratin layer, often exfoliating into the lumen. The basal layer shows nuclear polarization, with a flat junction with the connective tissue. ${ }^{13,14}$ Thus, lesions with these characteristic features would be diagnosed as DC-simple type when located outside the jaws, but diagnosed as OKC within jawbones. Only when the lining contains skin appendages, DC-compound type can be considered an appropriate diagnosis for an intraosseous lesion.

DC is considered a congenital hamartoma in soft tissues; whereas, $\mathrm{OKC}$ is now considered a neoplasm, and the name keratocystic odontogenic tumor (KOT) suggested by the WHO. ${ }^{13}$ The information from the cases of IDC indicate they have a significantly more indolent behavior than OKC. Both lesions have a lining of keratinizing epithelium, but only IDC present sebaceous glands, and possibly also sweat glands in the cyst lining- feature that help differentiate between the two.

The impaction of both molars within the lesion in our patient indicates the cyst developed prior to the physiologic eruption time of the second molar (at around age 12). Thus, we deduce the lesion was present for 45 years or more.

The apparently slow growth together with absence of recurrence in previous cases indicate an indolent biological behavior for IDC, as opposed to OKC which is an aggressive lesion with a high recurrence rate ${ }^{14}$ (Clinical radiographic and microscopic characteristics of OKC and IDC are summarized in Table 1).

In conclusion, IDC of the jaws is a rare nonaggressive lesion. It shares some microscopic features with $\mathrm{OKC}$, but can be distinguished by the presence of sebaceous glands only in IDC.

Table 1: Comparison between odontogenic keratocyst and Intraosseous dermoid cyst of the jaws

\begin{tabular}{|lll|}
\hline & OKC & IDC \\
\hline Age & Wide range & Wide range \\
Mandible: Maxilla & $3: 1$ & $1: 1$ \\
Radiographic characteristics & Uni or multilocular & Unilocular \\
Expansion, swelling, pain & $82 \%$ & $21.4 \%$ \\
Impacted/displaced teeth & $37 \%$ & $21.4 \%$ \\
Cortical perforation & common & rare \\
Rate of recurrence & $\sim 30 \%$ & $0 \%$ \\
\hline
\end{tabular}




\section{REFERENCES}

1. Bodner L, Woldenberg Y, Sion-Vardy N. Dermoid cyst of the maxilla. Int J Oral Maxillofac Surg 2005;34:453-55.

2. Al-Khateeb TH, Al-Masri NM, Al-Zoubi F. Cutaneous cysts of the head and neck. J Oral Maxillofac Surg 2009;67(1):52-57.

3. Takeda Y, Oikawa Y, Satoh M, Nakamura S. Latent form of multiple dermoid cysts in the jaw bone. Pathol Int 2003;53: 786-89.

4. Komiyama K, Miki Y, Oda Y, Tachibana T, Okaue M, Tanaka $\mathrm{H}$, Moro I. Uncommon dermoid cyst presented in the mandible possibly originating from embryonic epithelial remnants. J Oral Pathol Med 2002;31:184-87.

5. Oji C. Statistical observations on jaw cysts in Enugu, Nigeria, 1987-1996. Odontostomatol Trop 1999;22:33-36.

6. Vuhahula E, Nikai H, Ijuhin N, Ogawa I, Takata T, Koseki T, Tanimoto K. Jaw cysts with orthokeratinization: Analysis of 12 cases. J Oral Pathol Med 1993;22:35-40.

7. Lindh C, Larsson A. Unusual jaw-bone cysts. J Oral Maxillofac Surg 1990;48:258-63.
8. Craig GT, Holland CS, Hindle MO. Dermoid cyst of the mandible. Br J Oral Surg 1980;18:230-37.

9. Brannon RB. The odontogenic keratocyst. A clinicopathologic study of 312 cases. Part II. Histologic features. Oral Surg Oral Med Oral Path 1977;43:233-55.

10. Issa MA, Davis JD. Dermoid cyst of the jaws. Brit Dent J 1971;131:543-46.

11. Dudani IC, Khanna JN. Dermoid inclusion cyst of the maxilla. J Indian Dent Assoc 1971;43:188-91.

12. Halperin C, Kolas S, Jefferis KR, et al. Occurrence of Fordyce spots, benign migratory glossitis and fissured tongue in 2478 dental patients. Oral Surg Oral Med Oral Pathol 1953;9:1072.

13. Barnes L, Eveson JW, Reichart P, et al (Eds). World health organization classification of tumors. Pathology and genetics of head and neck tumors. Lyon: IARC Press 2005.

14. Shear M, Speight PM (Eds). Cysts of the oral and maxillofacial regions (4th ed). Oxford: Blackwell Munksgaard 2007. 